$X$. D. Li, V. Trimble, and Z. R. Wang, eds.

\title{
New Chandra Results on Seyfert I Galaxies: Fe-K lines
}

\author{
Urmila Padmanabhan ${ }^{1}$ and Tahir Yaqoob ${ }^{1,2}$ \\ 1 Johns Hopkins University, 3400 N. Charles St., Baltimore, MD 21218, \\ USA \\ ${ }^{2}$ NASA/GSFC, Code 662, Greenbelt Rd., Greenbelt, MD 20771, USA
}

\begin{abstract}
We present measurements of the Fe-K $\alpha$ line for nine Seyfert I galaxies using Chandra High Energy Transmission Grating Spectrometer (HETGS) data. The centroid energies are narrowly dispersed (6.403 \pm $0.062 \mathrm{keV}$ ) and indicate an origin of the line cores in cold matter. If all the lines in this sample were the peaks of a relativistically broadened disk line, it would require unrealistically fine tuning. However, at least three of the nine AGN clearly show a complex Fe-K $\alpha$ line with an underlying broad component, possibly from a disk. In the others, an apparently narrow Fe-K $\alpha$ line (even if it is resolved by Chandra) may still be due to the peak of a disk line. To distinguish this scenario from an origin in distant matter requires variability information.
\end{abstract}

\section{Introduction}

The Fe-K $\alpha$ emission lines in type 1 AGN can be complex, consisting of a broad $(\sim 10,000-100,000 \mathrm{~km} / \mathrm{s}$ FWHM $)$ and/or a narrow $(<10,000 \mathrm{~km} / \mathrm{s} \mathrm{FWHM})$ component (e.g. Yaqoob et al. 2002). The broad component probably originates from the accretion disk, having suffered severe Doppler and gravitational energy shifts in the inner disk (e.g. see review by Fabian et al. 2000) and has been clearly observed by $A S C A$. Chandra and $X M M$ are revealing that a narrow-line (NL) component is quite common in type 1 AGN (Reeves et al. 2001; Yaqoob et al. 2001a; Yaqoob et al. 2001b; Kaspi et al. 2001; Pounds et al. 2001; Turner et al. 2002, Fang et al. 2002), often superimposed on top of a broad component. Even with Chandra, the NL component has only been resolved in two cases at $>99 \%$ confidence (NGC 3783 [Kaspi et al. 2002] and MCG -6-30-15 [Lee et al. 2002]). Interpreting the line width in NGC $3783(1720 \pm 360 \mathrm{~km} / \mathrm{s}$ FWHM) as the result of Doppler shifts in a spherical gas distribution with Keplerian velocity places its origin in between the BLR and NLR. However, variability information is required to truly constrain the origin of the line. The NL observed in MCG $-6-30-15$ has a width of $\sim 3600 \mathrm{~km} / \mathrm{s}$ FWHM (high flux state) but it cannot arise from distant matter, as demonstrated by the rapid variability of the line (Lee et al. 2002). Thus, some of the narrow lines observed by Chandra could be the cores of an underlying broad disk line.

Here we examine the data from nine Seyfert I galaxies observed by the Chandra HETGS. We present the most precise measurements to date of the peak energies and widths of the line cores. By comparing the confidence contours of the equivalent widths (EW) versus FWHM velocity widths and line intensity 
versus peak energy, we can infer important differences about the origin of the lines in different sources.

\section{Method}

The analysis method was similar to that used in Yaqoob et al. (2001a) except that the data were reprocessed using ciao 2.1.3 and CALDB version 2.7, according to recipes described in ciao 2.1.3. The HETGS is composed of the High Energy Grating (HEG) and the Medium Energy Grating (MEG) and we used only the HEG data since it has the best energy resolution $(1860 \mathrm{~km} / \mathrm{s}$ FWHM at $6.4 \mathrm{keV}$ ) in the Fe-K $\alpha$ region. We fitted the HEG data with a model of simple power law plus a Gaussian from $2 \mathrm{keV}$ to $7 \mathrm{keV}$. All parameters, including the line width, were allowed to float. The power law slope was then frozen at the best-fit value. Using just the 5 to $7 \mathrm{keV}$ data, the joint confidence contour plots of the line intensity versus the line energy, and EW versus the FWHM were made. These are shown in Figures 1 and 2 respectively. The best-fitting line parameters, with statistical errors at $90 \%$ confidence for one interesting parameter, are shown in Table 1. Note that the results obtained when the power-law slope is allowed to float are similar (full details will be presented elsewhere).

Table 1. Power law plus Gaussian fits to the Chandra (HEG) data.

\begin{tabular}{|c|c|c|c|c|}
\hline Source & $\begin{array}{c}\mathrm{E}^{\mathrm{a}} \\
(\mathrm{keV})\end{array}$ & $\begin{array}{c}\mathrm{I}^{\mathrm{b}} \\
\left(10^{-5} \text { photons } \mathrm{cm}^{-2} \mathrm{~s}^{-1}\right)\end{array}$ & $\begin{array}{l}\mathrm{EW}^{\mathrm{c}} \\
(\mathrm{eV})\end{array}$ & $\begin{array}{c}\text { FWHM } \\
(\mathrm{km} / \mathrm{s})\end{array}$ \\
\hline NGC 3516 & $6.400_{-0.010}^{+0.008}$ & $4.3_{-1.1}^{+1.3}$ & $162_{-41}^{+47}$ & $1390_{-1390}^{+1640}$ \\
\hline NGC 3783 & $6.399_{-0.013}^{+0.013}$ & $5.3_{-1.8}^{+2.2}$ & $79_{-27}^{+33}$ & $2640_{-1265}^{+2200}$ \\
\hline NGC 5548 & $6.397_{-0.022}^{+0.020}$ & $3.0_{-1.3}^{+1.5}$ & $106_{-46}^{+53}$ & $3650_{-2000}^{+2690}$ \\
\hline $3 \mathrm{C} 120$ & $6.414_{-0.016}^{+0.019}$ & $3.0_{-1.4}^{+1.8}$ & $61_{-29}^{+366}$ & $2090_{-1920}^{+2860}$ \\
\hline Mkn 509 & $6.431_{-0.023}^{+0.027}$ & $2.8_{-1.7}^{+1.9}$ & $45_{-27}^{+30}$ & $2740_{-2740}^{+2630}$ \\
\hline NGC 4593 & $6.402_{-0.020}^{+0.014}$ & $3.2_{-1.3}^{+1.5}$ & $73_{-29}^{+355}$ & $2140_{-1480}^{+4030}$ \\
\hline NGC 4051 & $6.417_{-c}^{+}$ & $2.7_{-1.3}^{+1.6}$ & $147_{-69}^{+86}$ & $5190_{-3540}^{+5530}$ \\
\hline F 9 & $6.424_{-}^{+}$ & $2.2_{-1 .}^{+2.9}$ & $86_{-43}^{+113}$ & $4040_{-1840}^{+1570}$ \\
\hline $3 \mathrm{C} 273$ & $6.348_{-0.048}^{+0.052}$ & $5.5_{-1.5}^{+5.9}$ & $32_{-9}^{+35}$ & $4610_{-4170}^{+8050}$ \\
\hline
\end{tabular}

$\overline{\text { Statistical errors are for } 90 \% \text { confidence for one interesting parameter }(\Delta C}=$ 2.706). ${ }^{a}$ Center energy of Gaussian line. ${ }^{b}$ Line Intensity. ${ }^{c}$ Equivalent Width.

\section{Centroid Energies of the Fe-K lines}

In Figure 1 we show the line intensity versus line center energy confidence contours. Note that the dotted line is at $6.4 \mathrm{keV}$ and all the contours intersect it, at least at the $99 \%$ confidence level. Due to the superb energy resolution of the Chandra HEG, the $90 \%$ statistical errors on the line energy can be as small as $10 \mathrm{eV}$. The most tightly constrained contours are those of NGC 3516, which has an exposure time of $100 \mathrm{ks}$ and a comparatively low $(2-10 \mathrm{keV})$ flux of $2.5 \times 10^{-11} \mathrm{erg} \mathrm{cm}^{-2} \mathrm{~s}^{-1}$. The weighted mean centroid energy of the line detected in the nine Seyfert galaxies is $6.403 \pm 0.062 \mathrm{keV}$. The small dispersion is quite remarkable. If all these lines were from a disk, the disk parameters would 


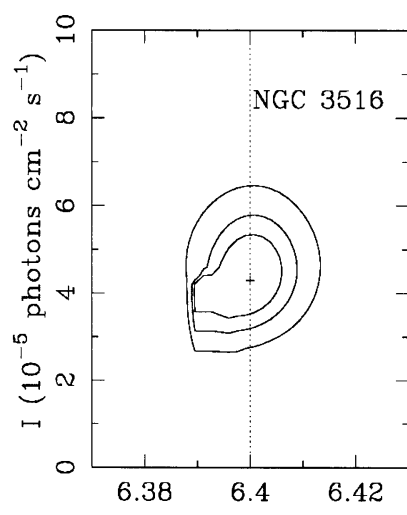

$\mathrm{E}(\mathrm{keV})$

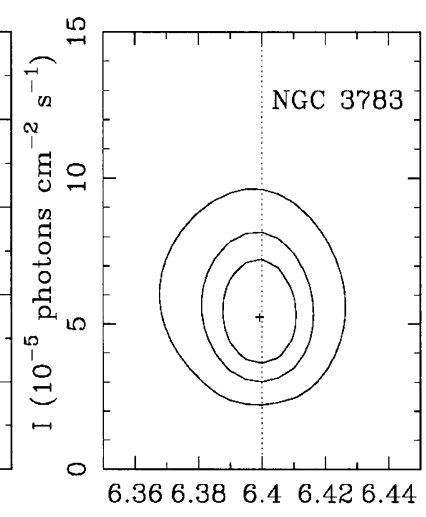

$\mathrm{E}(\mathrm{keV})$

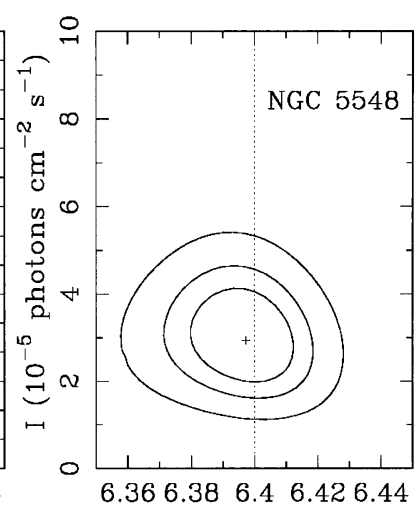

$\mathrm{E}(\mathrm{keV})$
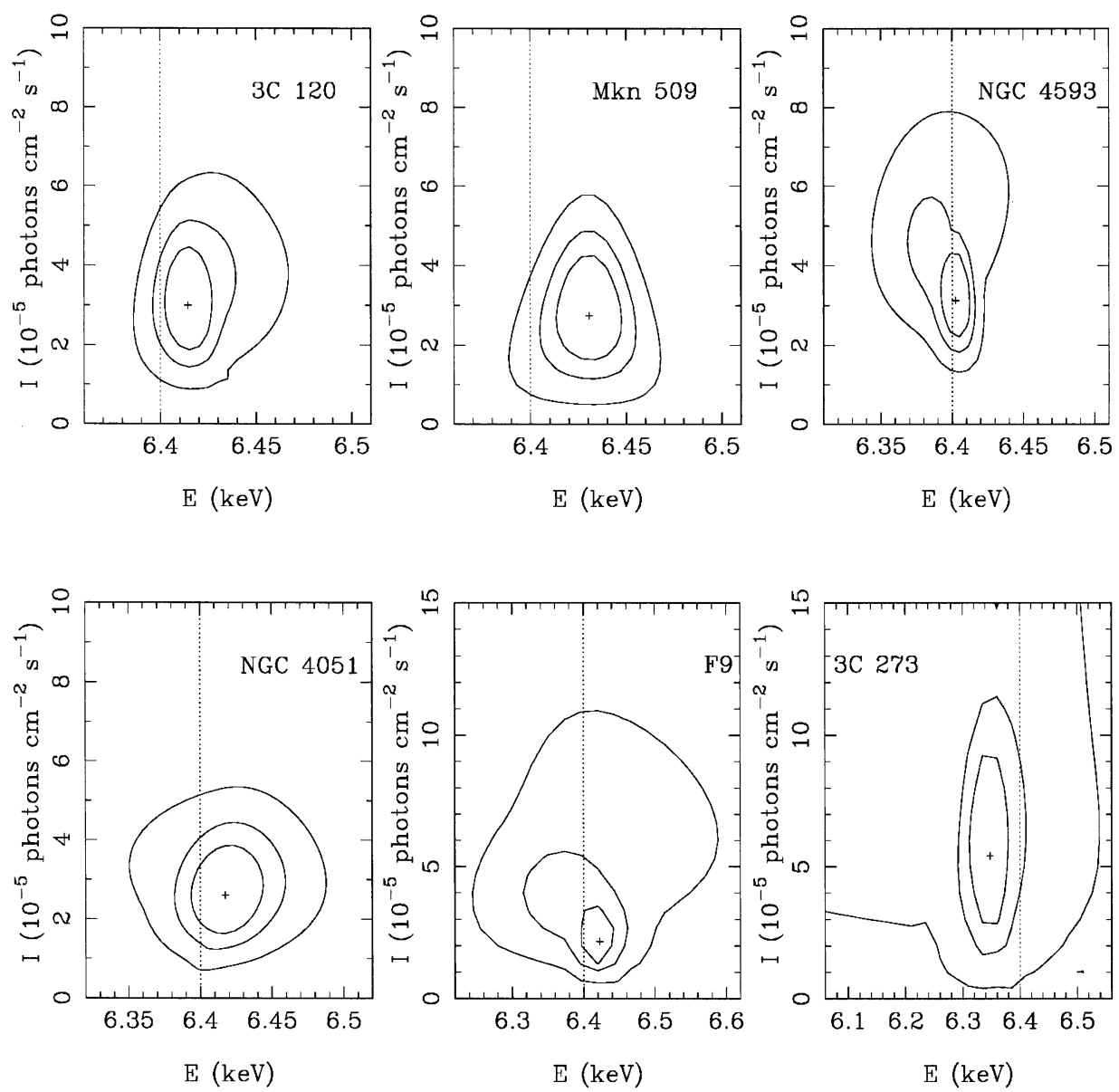

Figure 1. HEG confidence contours $(68 \%, 90 \%, 99 \%)$ of $\mathrm{Fe}-\mathrm{K}$ line of Line Intensity versus Line Energy for the nine AGN. 
need to be finely tuned. Whatever the origin of the lines, the cores observed by the HEG are formed in cold Fe.

The detailed shapes of the contours carry additional information about the line profiles. Comparing the contours of NGC 5548 and F 9, we see a clear and marked difference in the shapes of their contours. However, they have a similar signal-to-noise ratio (SNR) as evidenced by the number of photons in the 5 to 7 $\mathrm{keV}$ band. Thus, the difference in the contours are due to the intrinsic differences in their line profiles and are not due to SNR effects. The broader contours of F 9 indicate a complex line, consistent with the line profile in Yaqoob et al. (2001b). $R X T E$ data, simultaneous with the Chandra observation for $\mathrm{F} 9$, confirms the presence of a broad He-like Fe-K $\alpha$ component (see Yaqoob \& Padmanabhan 2003; hereafter YP03). The SNR in the other seven AGN is greater than that of NGC 5548 and F 9. Therefore, we can infer that the differences in the contours of all the AGN are not due to SNR effects, except for 3C 273 where the EW of the line is much less than in the other sources. The contours of NGC 3516, NGC 3783, 3C 120 and MKN 509 are very similar to that of NGC 5548 and those of NGC 4051 and NGC 4593 are similar to that of F 9.

\section{EW versus FWHM}

In Figure 2 the EW versus FWHM confidence contours are shown. Note that the dotted line on these plots shows the FWHM resolution of the HEG (1860 $\mathrm{km} / \mathrm{s}$ at $6.4 \mathrm{keV}$ for $z=0$ ) at the measured, observed peak energy of each line (that is, accounting for redshift). Again, comparing NGC 5548 and F 9 (since they have similar SNR), we find that their contours have very different shapes. The contours of NGC 5548 show the $99 \%$ confidence level (CL) FWHM bound at $\sim 10,000 \mathrm{~km} / \mathrm{s}$ with the line peak unresolved at the $99 \% \mathrm{CL}$. On the other hand, in F 9 the FWHM extends out $\sim 60,000 \mathrm{~km} / \mathrm{s}$ at the $99 \%$ CL (which is off the scale in Figure 2 but can be seen in YP03). F 9 also shows the only resolved peak at the $99 \% \mathrm{CL}$ in this sample. Thus, the lines in F 9 and NGC 5548 are very clearly different, with the line in F 9 definitely accompanied by a broader component and the line in NGC 5548 comparatively narrower. The FWHM contours of NGC 3516, NGC 3783, 3C 120, and Mkn 509 are similar to that of NGC 5548. NGC 4593, NGC 4051 and F 9 all show clear underlying broad components. Except for F 9, all the Fe-K $\alpha$ lines in the sample, show an unresolved peak at the $99 \%$ CL.

Lee et al. (2002) found that in a Chandra observation of MCG -6-30-15, the HEG Fe-K $\alpha$ line was resolved, with a best-fit FWHM width of $11,000 \mathrm{~km} / \mathrm{s}$ (time-averaged). Considering only the high flux state, the line was resolved with a best-fit FWHM width of $3600 \mathrm{~km} / \mathrm{s}$. However, this line was shown to be variable on short time scales (hundreds of seconds). Thus, this line is most probably the core of a very peaky disk line, since it is impossible for either the putative obscuring torus, or even the BLR to respond to continuum variations so quickly. This demonstrates that even the AGN in our sample which show $99 \%$ CL FWHM contours less than $15,000 \mathrm{~km} / \mathrm{s}$ may have $\mathrm{Fe}-\mathrm{K} \alpha$ line cores which are from a disk and not from more distant matter.

On the other hand, for the dispersion in the line centroid energies to be so small, the radial emissivity of the disk must be very flat and the disk inclination 

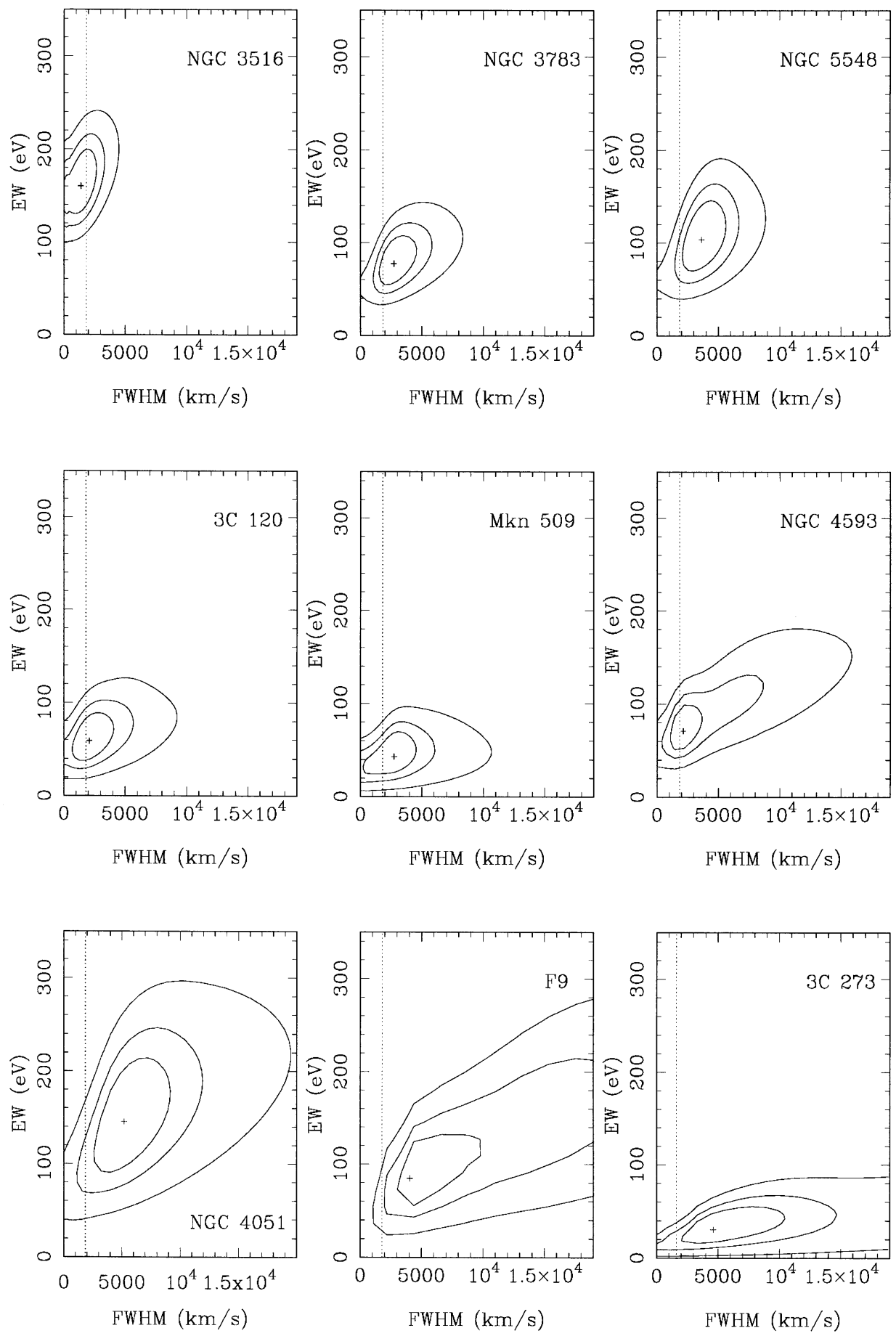

Figure 2. HEG confidence contours $(68 \%, 90 \%, 99 \%)$ of Fe-K line EW vs. FWHM of the nine AGN. 
angles must be small and narrowly distributed (almost face-on). To determine the dominant contribution to the line core (disk or distant matter) requires variability information. The EWs of the line cores range from $\sim 30 \mathrm{eV}$ to $\sim 150$ $\mathrm{eV}$. When there is an additional broad line, as measured by a higher throughput mission (e.g. RXTE; see YP03), the total EW can easily exceed twice this value. However, an interpretation of the EWs requires detailed physical modeling which will be presented elsewhere.

\section{Conclusions}

Measurements of the Fe-K $\alpha$ line centroid energy and width for nine Seyfert I galaxies using the best energy resolution available show that the centroid energies of the line cores are narrowly dispersed $(6.403 \pm 0.062 \mathrm{keV})$ and are consistent with a dominant contribution to the line core from cold matter. Three sources (NGC 4051, NGC 4593 and F 9) clearly show a complex Fe-K $\alpha$ line with an underlying broad, relativistic component. In the remaining AGN (except 3C $273)$, although the FWHM is $<11,000 \mathrm{~km} / \mathrm{s}(99 \% \mathrm{CL})$, we cannot rule out the line core still coming from a disk (as opposed to originating in distant matter). However, the small dispersion in the peak energy requires a very flat radial emissivity law and a narrowly distributed disk inclination angle. Variability information is required to determine the origin of the line core in these cases.

Acknowledgments. The authors acknowledge support from NASA grants NCC-5447 (T.Y., U.P.), NAG5-10769 (T.Y.), and CXO grants GO1-2101X, GO1-2102X (T.Y.). The authors are grateful to the Chandra and RXTE instrument and operations teams for their work. We also thank our collaborators, B. McKernan, I. M. George, T. J. Turner, \& K. Weaver.

\section{References}

Fabian, A. C., Iwasawa, K., Reynolds, C. S., \& Young, A. J. 2000, PASP, 112, 1145

Fang, T. et al.. 2002, ApJ, 565, 86

Kaspi, S. et al. 2002, ApJ, 554, 216

Lee, J. C. et al. 2002, ApJ, 570, L47

Reeves, J. N. et al. 2001, A\&A, 365, L134

Turner, T. J. et al. 2002, ApJ, 574, L123

Pounds, K. et al. 2001, ApJ, 559, 181

Yaqoob, T. et al. 2001a, ApJ, 546, 759

Yaqoob, T. \& Padmanabhan, U. 2003, in ASP Conf. Ser., Active Galactic Nuclei, from Central Engine to Host Galaxy, ed. S Collin, F. Combes \& I. Shlosman, (San Francisco: ASP), p. 7 (astro-ph/0211349) [YP03]

Yaqoob, T. et al. 2001b, in Conf. Proc., X-ray Emission from Accretion onto Black Holes, Proceedings, ed. T. Yaqoob, \& J. H. Krolik (published electronically on ADS), E79 (astro-ph/0111419)

Yaqoob, T., Padmanabhan, U., Dotani, T., \& Nandra, K. 2002, ApJ, 569, 487 